\title{
Targeting PPM1D by lentivirus-mediated RNA interference inhibits the tumorigenicity of bladder cancer cells
}

\author{
W. Wang ${ }^{1,3}$, H. Zhu ${ }^{3}$, H. Zhang ${ }^{2}$, L. Zhang ${ }^{2}$, Q. Ding ${ }^{1,2}$ and H. Jiang ${ }^{1,2}$ \\ ${ }^{1}$ Institute of Urology, Huashan Hospital, Fudan University, Shanghai, China \\ ${ }^{2}$ Department of Urology, Huashan Hospital, Fudan University, Shanghai, China \\ ${ }^{3}$ Department of the Intensive Care Unit, Huashan Hospital, Fudan University, Shanghai, China
}

\begin{abstract}
Protein phosphatase magnesium/manganese-dependent 1D (PPM1D) is a p53-induced phosphatase that functions as a negative regulator of stress response pathways and has oncogenic properties. However, the functional role of $P P M 1 D$ in bladder cancer $(B C)$ remains largely unknown. In the present study, lentivirus vectors carrying small hairpin RNA (shRNA) targeting PPM1D were used to explore the effects of PPM1D knockdown on BC cell proliferation and tumorigenesis. shRNAmediated knockdown of PPM1D significantly inhibited cell growth and colony forming ability in the BC cell lines 5637 and T24. Flow cytometric analysis showed that PPM1D silencing increased the proportion of cells in the G0/G1 phase. Downregulation of PPM1D also inhibited 5637 cell tumorigenicity in nude mice. The results of the present study suggest that PPM1D plays a potentially important role in BC tumorigenicity, and lentivirus-mediated delivery of shRNA against PPM1D might be a promising therapeutic strategy for the treatment of BC.
\end{abstract}

Key words: Protein phosphatase magnesium/manganese-dependent 1D; Bladder cancer; Gene silencing; RNA interference; Proliferation

\section{Introduction}

In 2012, bladder cancer (BC) was the fourth most common cancer in males and the eighth most common in females in the United States (1). Approximately $20 \%$ of tumor node metastasis (TNM) stage T1 primary tumors that undergo re-resection progress to invasive $\mathrm{BC}(2,3)$. In addition, overexpression of p53, p21, and p16 is associated with increased risk of recurrence and poor long-term survival in $\mathrm{BC}$, suggesting that targeted treatment in the early stages of the disease could be a useful strategy $(4,5)$.

Protein phosphatase magnesium/manganese-dependent 1D (PPM1D), also called wild-type p53-induced phosphatase (Wip1), is a member of the magnesium-dependent serine/threonine protein phosphatase (PPM) family $(6,7)$. It was first identified as a phosphatase induced by p53 in response to ultraviolet and ionizing radiation (8). The $P P M 1 D$ gene is located on chromosome $17 q 23.2$ and is a negative regulator of stress response pathways. PPM1D plays a variety of roles in cellular processes, including abrogation of cell cycle checkpoints and inhibition of senescence, apoptosis, and DNA repair (9). Studies have shown that PPM1D possesses oncogenic properties $(10,11)$. Amplified levels of the PPM1D gene have been found in several cancer cell lines including neuroblastoma and lung, breast, pancreatic, bladder, and liver cancers $(11,12)$. Moreover, $P P M 1 D$ is overexpressed in a number of human primary tumors, such as breast cancer (13), ovarian cancer $(14,15)$, neuroblastoma (16), hepatocellular cancer (17) and lung cancer (18), and is associated with poor prognosis.

RNA interference (RNAi) is an endogenous protein suppression mechanism by which short double-stranded RNA (dsRNA) mediates sequence-specific degradation of mRNA, thereby preventing translation of the protein encoded by the target mRNA $(19,20)$. RNAi can be used to specifically target mutant genes, cancer-associated genes or receptors involved in oncogenic pathways, thereby opening new avenues in anticancer therapy $(21,22)$. RNAi has been successfully used to control cell proliferation and the invasive ability of $\mathrm{BC}$ cells (23).

To elucidate the role of PPM1D in BC, we used lentivirusdelivered shRNA to knock down PPM1D expression. This

Correspondence: H. Jiang, Department of Urology, Huashan Hospital, Fudan University, No. 12, WuLuMuQi Middle Road, Shanghai 200040, China. Fax: 86-21-6249-5490. E-mail: drjianghaowen@126.com 
model system was used to examine the effect of PPM1D silencing on $\mathrm{BC}$ cell proliferation and growth and the antitumor potential of PPM1D shRNA in vivo and in vitro.

\section{Material and Methods}

\section{Cells lines and cell culture}

The human urinary BC cell lines 5637 and T24 and the human renal epithelial cell line HEK293T were purchased from the American Type Culture Collection (USA) and maintained at $37^{\circ} \mathrm{C}$ and $5 \% \mathrm{CO}_{2}$. The HEK293T and T24 cell lines were cultured in Dulbecco's modified Eagle's medium (DMEM; Invitrogen, USA) supplemented with 10\% fetal bovine serum (FBS, Invitrogen), and the 5637 cell line was cultured in RPMI-1640 (Invitrogen) supplemented with $10 \%$ FBS.

\section{Lentiviral plasmid construction, lentivirus production, and cell infection}

The human PPM1D (Gen-Bank accession no. NM_003620.3) specific small interfering RNA (siRNA) sequence, which was designed with online software from Invitrogen, was 5'-CCCTTCTCGTGTTTGCTTAAA-3'. The nonsilencing (NS) sequence (5'-TTCTCCGAACGTGTCAC GT-3') was used as a scrambled control (24). Pairs of complementary oligonucleotides with these sequences were synthesized, annealed, and ligated into a linearized pGCSIL-GFP plasmid vector. These plasmids were amplified in E. coli DH5 and purified using a QIAGEN Plasmid Maxi Kit (Qiagen, The Netherlands). Lentivirus was generated in 293T cells by cotransfection of the recombinant pGCSIL-GFP vector, together with pHelper 1.0 and pHelper 2.0 plasmids using Lipofectamine 2000 (Invitrogen). The lentiviral particles were harvested $48 \mathrm{~h}$ after transfection and purified by ultracentrifugation ( $2 \mathrm{~h}$ at $50,000 \mathrm{~g}$ ) (25), and are hereafter referred to as LV-si-PPM1D (a specific interference construct for PPM1D) or LV-si-CTRL (negative control). For cell infection, 30\% confluent 5637 and T24 cells were incubated with lentiviruses for $48 \mathrm{~h}$, and the medium, which contained puromycin $(10 \mu \mathrm{g} / \mathrm{mL}$; Sigma-Aldrich, USA), was replaced to select stable clones. Each cell line was divided into two experimental groups, the si-CTRL group (cells infected with Lv-siCTRL) and the si-PPM1D group (cells infected with LV-siPPM1D).

\section{Quantitative real-time polymerase chain reaction (PCR) and Western blotting}

Total RNA was extracted and reverse-transcribed as described previously (26). Quantitative real-time PCR reactions were carried out with an $A B I$ Prism 7900 Sequence Detection System (PE Applied Biosystems, USA) using $25 \mu \mathrm{L}$ of a reaction mixture that consisted of $0.1 \mu \mathrm{M}$ primers, $10 \mu \mathrm{L} 2 \times$ SYBR Premix Ex Taq (Takara, Japan), and 20-100 ng cDNA sample. The following primers were used: PPM1D, 5'-AGAGAATGTCCAAGGTGTAGTC
-3' and 5'-TCGTCTATGCTTCTTCATCAGG-3'; $\beta$-actin, 5'-GTGGACATCCGCAAAGAC-3' and 5'-TCGTCTATGCT TCTTCATCAGG-3'. An initial denaturation/activation step $\left(15 \mathrm{~s}, 95^{\circ} \mathrm{C}\right)$ was followed by 45 cycles $\left(5 \mathrm{~s}\right.$ at $95^{\circ} \mathrm{C}, 30 \mathrm{~s}$ at $\left.60^{\circ} \mathrm{C}\right)$. The relative expression of $P P M 1 D$ mRNA was calculated with the $2^{-\Delta \Delta \mathrm{Ct}}$ method, and $\beta$-actin mRNA expression was used for normalization.

Western blot analysis was performed to detect PPM1D protein expression. Cells were scraped and homogenized in radioimmunoprecipitation assay (RIPA) lysis buffer. Proteins extracted from cellular lysates were separated on $12 \%$ sodium dodecyl (SDS)-polyacrylamide gels and transferred onto polyvinylidene fluoride (PVDF) membranes (Millipore, USA). After blocking, the membranes were incubated with mouse anti-PPM1D and anti-GAPDH monoclonal antibodies $(1: 200$ and $1: 5000$, respectively, Santa Cruz Biotechnology, USA) overnight at $4^{\circ} \mathrm{C}$. After washing with Tris-buffered saline/Tween-20 solution, the membranes were incubated with horseradish peroxidaseconjugated goat anti-mouse IgG (1:5000, Santa Cruz Biotechnology) at room temperature for $1 \mathrm{~h}$. Bands were detected using an enhanced chemiluminescence system (Amersham, USA).

\section{Cell proliferation and colony formation assay}

Cells were trypsinized, resuspended, seeded onto 96well plates in $100 \mu \mathrm{L}\left(5 \times 10^{3}\right.$ cells $)$ per well, and incubated at $37^{\circ} \mathrm{C}$. The number of viable cells was measured daily using 3-(4,5-dimethylthiazol-2-yl)-2,5-diphenyltetrazolium bromide (MTT) as described previously (26). For the cell colony formation assay, cells were seeded onto 6 -well plates at a density of 200 cells per well and cultured at $37^{\circ} \mathrm{C}$ for 14 days. After fixing with paraformaldehyde, cells were stained with Giemsa (Sigma) for $10 \mathrm{~min}$, and washed with double distilled $\mathrm{H}_{2} \mathrm{O}$ three times. The plates were photographed with a digital camera. Each experiment was performed in triplicate and repeated three times.

\section{Flow cytometric assay}

Cells were harvested and fixed with cold $70 \%$ ethanol for $1 \mathrm{~h}$. The cells were sequentially centrifuged ( $5 \mathrm{~min}$ at $100 \mathrm{~g}$ ) and resuspended with phosphate-buffered saline (PBS). Cells were stained with propidium iodide (Sigma-Aldrich) at $4^{\circ} \mathrm{C}$ for $30 \mathrm{~min}$ in the dark and analyzed using flow cytometry. Each experiment was conducted in triplicate.

\section{Animal experiments}

Five-week-old male BALB/c mice were purchased from Shanghai Slac Laboratory Animal Co. Ltd. (China) and received humane care in compliance with the Guidelines for the Care and Use of Experimental Animals in Research. Mice were divided into 2 groups of 10 mice each, referred to as the si-CTRL and si-PPM1D groups. A total of $5 \times 10^{6}$ Lv-si-CTRL or Lv-si-PPM1D infected cells were suspended in Eagle's minimal essential medium (EMEM) and injected subcutaneously into the right flank of each mouse in the 
corresponding group. The tumor diameter was measured, and the volume was calculated using the formula $\mathrm{V}=$ $0.4 \times \mathrm{ab}^{2} \quad(\mathrm{~V}=$ volume, $\mathrm{a}=$ largest diameter, $\mathrm{b}=$ smallest diameter) on days $10,14,18$, and 24 . Mice were photographed and humanely killed on day 24 , and the tumors were dissected and weighed.

\section{Statistical analysis}

All data are reported as means \pm SE. Statistical analysis was performed using the Student two-tailed unpaired $t$-test for comparisons between two groups. In all cases, $\mathrm{P}<0.05$ was considered to be statistically significant.

\section{Results}

\section{Lentivirus-mediated shRNA inhibited the expression of PPM1D in BC cells}

The 5637 and T24 cell lines were infected with Lvsi-PPM1D; the highest infection efficiency was $>90 \%$, as determined by detecting the expression of green fluorescent protein (GFP) $96 \mathrm{~h}$ after infection (Figure 1A). Quantitative real-time PCR analysis showed that the PPM1D mRNA level was significantly lower in the si-PPM1D group compared to the si-CTRL group (Figure 1B). The protein level of $P P M 1 D$ in the si-PPM1D group was also strongly decreased compared with the si-CTRL group (Figure 1C).

\section{Knockdown of PPM1D inhibited BC cell growth}

To examine the effect of PPM1D knockdown on $\mathrm{BC}$ cell
A
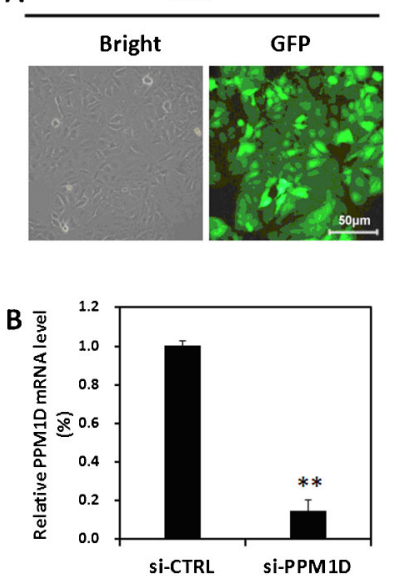

A

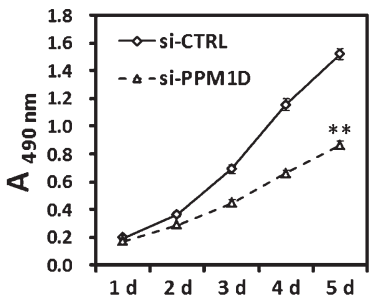

B

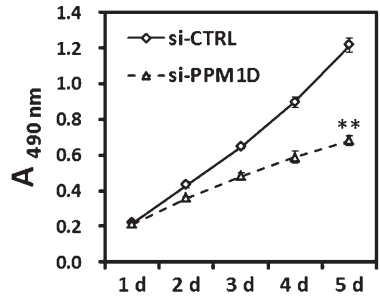

Figure 2. Knockdown of PPM1D attenuated the growth potential of bladder cancer cells in vitro. The proliferation of $5637(A)$ and T24 $(B)$ cells was assessed by MTT assay after infection with Lvsi-CTRL or LV-si-PPM1D. Data are reported as means \pm SD of three independent experiments. Lv: lentiviral; $d$ : day. ${ }^{* *} \mathrm{P}<0.01$, compared to Lv-si-CTRL ( $t$-test).

growth, Lv-si-PPM1D- or Lv-si-CTRL-infected 5637 and T24 cells were subjected to MTT and colony formation assays. As shown in Figure 2, cell proliferation in the si$P P M 1 D$ group was significantly inhibited compared with that in the si-CTRL group. Colony formation ability was significantly lower in the si-PPM1D group compared to the si-CTRL group (Figure 3). Similar results were obtained in 5637 and T24 cells.

Flow cytometric analysis showed that the proportion of cells in the G1 phase was markedly increased in the siPPM1D group compared with the si-CTRL group (Figure 4), partly explaining the growth suppression mediated by Lv-si$P P M 1 D$

\section{Knockdown of PPM1D inhibited BC tumorigenicity in vivo}

The 5637 cells infected with Lv-si-CTRL or Lv-si-PPM1D were subcutaneously implanted into nude mice to examine
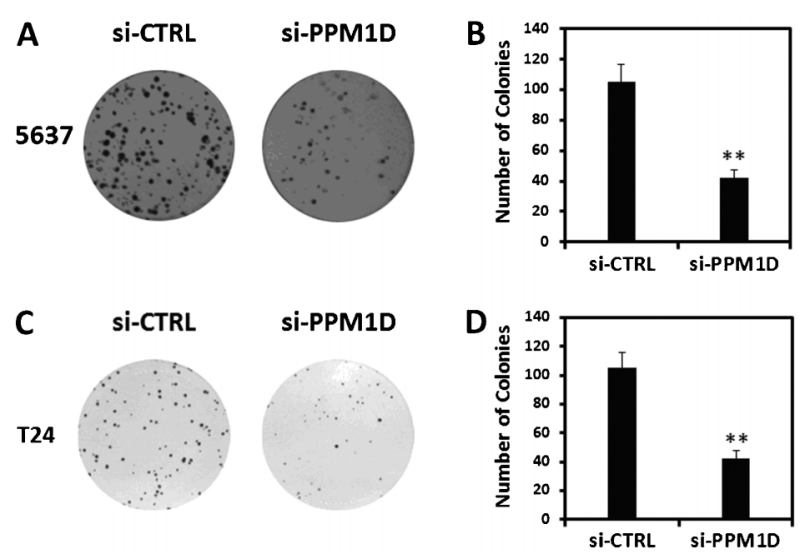

Figure 3. PPM1D knockdown decreased the colony formation ability of $5637(A, B)$ and T24 $(C, D)$ cells. Data are reported as means \pm SD of three independent experiments. The number of colonies was significantly different between cells infected with Lv-si-CTRL and with Lv-si-PPM1D ( ${ }^{* *} \mathrm{P}<0.01, t$-test). 

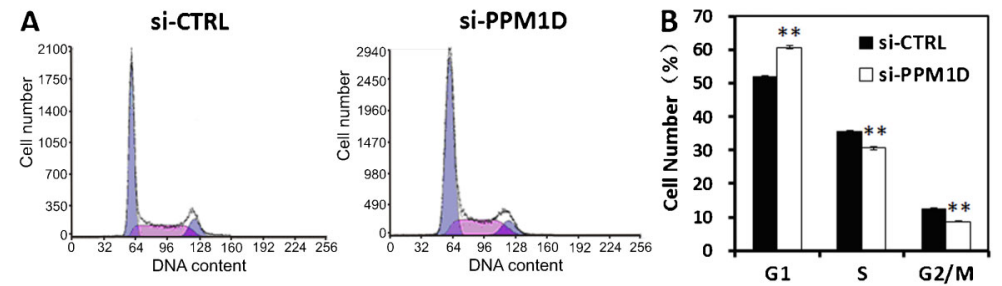

Figure 4. Knockdown of PPM1D increases the proportion of 5637 cells in G1 phase. Cell cycle distribution was analyzed by flow cytometry. $A$, Representative images of three independent FACS analyses are shown. B. Proportion of cells in the different cell cycle phases. Data are reported as means $\pm S D$ of three independent experiments. ${ }^{* *} \mathrm{P}<0.01$, compared to si-CTRL ( $t$-test). the effect of PPM1D knockdown on BC tumorigenicity in vivo. All of the mice in the si-CTRL group displayed steadily and progressively growing tumors, whereas the BC cells in the si-PPM1D group showed weaker tumorigenicity, and the mice developed smaller tumors (Figure 5).

\section{Discussion}

Gene dysregulation is frequently observed in cancer, and gene expression profiles vary among different cancers. $P P M 1 D$ or Wip1 is a serine/threonine phosphatase that is overexpressed and shows oncogenic activity in multiple human cancers (10). However, the role of the PPM1D gene in $\mathrm{BC}$ has not been investigated to date. To elucidate the function of $P P M 1 D$ in $B C$, we used lentivirus-mediated RNAi to inhibit PPM1D expression in T24 and 5637 cells and investigated the effects of $P P M 1 D$ knockdown in these $\mathrm{BC}$ cell lines. A lentiviral vector carrying PPM1D shRNA and a GFP reporter gene was constructed, which showed high infection efficiency in 5637 and T24 cells and effectively silenced PPM1D expression. These results indicated the successful construction of an effective shRNA vector targeting the $P P M 1 D$ gene.

Lentivirus-mediated PPM1D silencing strongly inhibited the growth and proliferation of T24 and 5637 BC cells in vitro, as demonstrated by MTT and colony formation assays. The data showed that the role of $P P M 1 D$ in $B C$ was consistent with that in other cancers $(16,27-29)$. Flow cytometric cell cycle analysis showed that PPM1D knockdown increased the proportion of T24 and 5637 BC cells in the G0/G1 phase, indicating that PPM1D downregulation blocked cell cycle progression. This could be a mechanism by which PPM1D silencing suppresses proliferation.

To determine the therapeutic value of lentivirusmediated RNAi of PPM1D for BC treatment, we analyzed its effect in a xenograft model. The results showed that sh-PPM1D lentivirus inhibited 5637 BC cell proliferation and suppressed their tumorigenic potential, indicating that targeting PPM1D may be a potential therapeutic strategy for the treatment of $B C$.

Previous studies have shown that PPM1D promotes tumorigenesis in a p53-dependent manner. $P P M 1 D$ is induced by p53 in response to various environmental stresses and facilitates the return of cells to the pre-stress state (6). In addition, PPM1D inhibits p53 activity by directly dephosphorylating p53 or its regulators such as ATM, Chk1, and Chk2, which indirectly inhibit p53 activity $(10,30)$. However, T24 and 5637 BC cells have p53 mutations, which implies that $P P M 1 D$ may function in a p53independent manner in BC cells. PPM1D is a target of p53 and other transcription factors, including the estrogen receptor- $\alpha$ and nuclear factor- $\kappa B(N F-\kappa B)(9)$. The p38

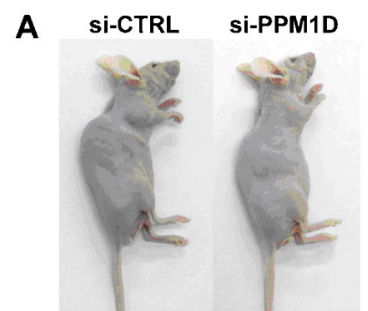

B

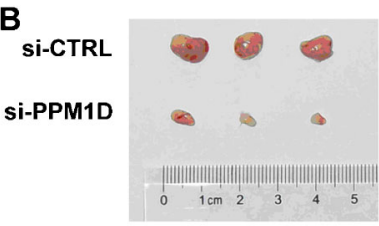

C
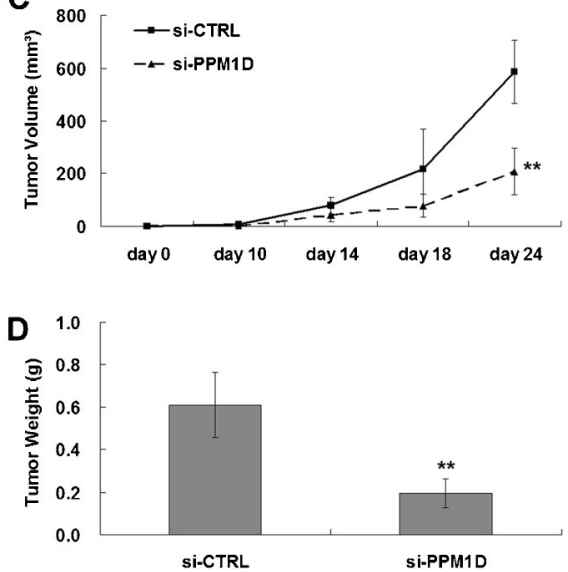

Figure 5. Knockdown of PPM1D inhibited the tumorigenicity of bladder cancer cells in vivo. Representative photographs of nude mice $(A)$ and tumors $(B)$ dissected from nude mice 24 days after injection of lentivirus-infected 5637 cells. C, Tumor volumes were recorded on days $10,14,18$, and 24 . $D$, Mice were humanely killed and tumors were weighed on day $24 .{ }^{* *} \mathrm{P}<0.01$, compared to si-CTRL ( $t$-test). 
mitogen-activated protein kinase (MAPK) (31) and Akt (28) signaling pathways may be downstream mediators of PPM1D activity in BC. Further studies are required to distinguish these mechanisms.

In conclusion, the results of the present study provided evidence that $P P M 1 D$ plays a potentially important role in BC tumorigenicity and could be a promising target for therapeutic intervention.

\section{References}

1. Siegel R, Naishadham D, Jemal A. Cancer statistics, 2013. CA Cancer J Clin 2013; 63: 11-30, doi: 10.3322/caac.21166.

2. Vianello A, Costantini E, Del Zingaro M, Bini V, Herr HW, Porena M. Repeated white light transurethral resection of the bladder in nonmuscle-invasive urothelial bladder cancers: systematic review and meta-analysis. J Endourol 2011; 25: 1703-1712, doi: 10.1089/end.2011.0081.

3. Ku JH, Lerner SP. Strategies to prevent progression of highrisk bladder cancer at initial diagnosis. Curr Opin Urol 2012; 22: 405-414, doi: 10.1097/MOU.0b013e328356adff.

4. Vishnu P, Mathew J, Tan WW. Current therapeutic strategies for invasive and metastatic bladder cancer. Onco Targets Ther 2011; 4: 97-113.

5. Gakis G, Schwentner C, Todenhofer T, Stenzl A. Current status of molecular markers for prognostication and outcome in invasive bladder cancer. BJU Int 2012; 110: 233237, doi: 10.1111/j.1464-410X.2011.10839.x.

6. Rossi M, Demidov ON, Anderson CW, Appella E, Mazur SJ. Induction of PPM1D following DNA-damaging treatments through a conserved p53 response element coincides with a shift in the use of transcription initiation sites. Nucleic Acids Res 2008; 36: 7168-7180, doi: 10.1093/nar/gkn888.

7. Douarre C, Mergui X, Sidibe A, Gomez D, Alberti P, Mailliet $P$, et al. DNA damage signaling induced by the G-quadruplex ligand 12459 is modulated by PPM1D/WIP1 phosphatase. Nucleic Acids Res 2013; 41: 3588-3599, doi: 10.1093/nar/ gkt073.

8. Fiscella M, Zhang H, Fan S, Sakaguchi K, Shen S, Mercer WE, et al. Wip1, a novel human protein phosphatase that is induced in response to ionizing radiation in a p53-dependent manner. Proc Natl Acad Sci U S A 1997; 94: 6048-6053, doi: 10.1073/pnas.94.12.6048.

9. Lowe J, Cha H, Lee MO, Mazur SJ, Appella E, Fornace AJ Jr. Regulation of the Wip1 phosphatase and its effects on the stress response. Front Biosci 2012; 17: 1480-1498, doi: $10.2741 / 3999$.

10. Lu X, Nguyen TA, Moon SH, Darlington $\mathrm{Y}$, Sommer M, Donehower LA. The type 2C phosphatase Wip1: an oncogenic regulator of tumor suppressor and DNA damage response pathways. Cancer Metastasis Rev 2008; 27: 123135, doi: 10.1007/s10555-008-9127-x.

11. Bulavin DV, Phillips $C$, Nannenga B, Timofeev O, Donehower $\mathrm{LA}$, Anderson CW, et al. Inactivation of the Wip1 phosphatase inhibits mammary tumorigenesis through p38 MAPKmediated activation of the p16(Ink4a)-p19(Arf) pathway. Nat Genet 2004; 36: 343-350, doi: 10.1038/ng1317.

12. Bulavin DV, Demidov ON, Saito $S$, Kauraniemi $P$, Phillips $C$, Amundson SA, et al. Amplification of PPM1D in human tumors abrogates p53 tumor-suppressor activity. Nat Genet

\section{Acknowledgments}

This research was supported by grants from the National Natural Science Foundation of China (Project No. 81272835), the Shanghai Science and Technology Commission and international cooperation projects (Project No. 11410708200), and the Shanghai Natural Science Foundation (Project No. 13ZR1405600).

2002; 31: 210-215, doi: 10.1038/ng894

13. Ruark E, Snape K, Humburg P, Loveday C, Bajrami I, Brough $\mathrm{R}$, et al. Mosaic PPM1D mutations are associated with predisposition to breast and ovarian cancer. Nature 2013; 493: 406-410, doi: 10.1038/nature11725.

14. Tan DS, Lambros MB, Rayter S, Natrajan R, Vatcheva R, Gao $Q$, et al. PPM1D is a potential therapeutic target in ovarian clear cell carcinomas. Clin Cancer Res 2009; 15: 2269-2280, doi: 10.1158/1078-0432.CCR-08-2403.

15. Ali AY, Abedini MR, Tsang BK. The oncogenic phosphatase PPM1D confers cisplatin resistance in ovarian carcinoma cells by attenuating checkpoint kinase 1 and p53 activation. Oncogene 2012; 31: 2175-2186, doi: 10.1038/onc.2011.399.

16. Saito-Ohara F, Imoto I, Inoue J, Hosoi H, Nakagawara A, Sugimoto $\mathrm{T}$, et al. $P P M 1 D$ is a potential target for $17 \mathrm{q}$ gain in neuroblastoma. Cancer Res 2003; 63: 1876-1883.

17. Li GB, Zhang XL, Yuan L, Jiao QQ, Liu DJ, Liu J. Protein phosphatase magnesium-dependent 1delta (PPM1D) mRNA expression is a prognosis marker for hepatocellular carcinoma. PLoS One 2013; 8: e60775, doi: 10.1371/journal. pone.0060775

18. Satoh N, Maniwa Y, Bermudez VP, Nishimura K, Nishio W, Yoshimura M, et al. Oncogenic phosphatase Wip1 is a novel prognostic marker for lung adenocarcinoma patient survival. Cancer Sci 2011; 102: 1101-1106, doi: 10.1111/j.1349-7006. 2011.01898.x.

19. Castanotto D, Rossi JJ. The promises and pitfalls of RNAinterference-based therapeutics. Nature 2009; 457: 426433, doi: 10.1038/nature07758.

20. Mohr SE, Perrimon N. RNAi screening: new approaches, understandings, and organisms. Wiley Interdiscip Rev RNA 2012; 3: 145-158, doi: 10.1002/wrna.110.

21. Bora RS, Gupta D, Mukkur TK, Saini KS. RNA interference therapeutics for cancer: challenges and opportunities (review). Mol Med Rep 2012; 6: 9-15.

22. Seth S, Johns R, Templin MV. Delivery and biodistribution of siRNA for cancer therapy: challenges and future prospects. Ther Deliv 2012; 3: 245-261, doi: 10.4155/tde.11.155.

23. Zhang $\mathrm{H}$, Jiang $\mathrm{H}$, Wang $\mathrm{W}$, Gong J, Zhang L, Chen Z, et al. Expression of Med19 in bladder cancer tissues and its role on bladder cancer cell growth. Urol Oncol 2012; 30: 920927, doi: 10.1016/j.urolonc.2010.10.003.

24. Pullmann R Jr, Juhaszova M, Lopez dS, I, Kawai T, MazanMamczarz K, Halushka MK, et al. Enhanced proliferation of cultured human vascular smooth muscle cells linked to increased function of RNA-binding protein HuR. J Biol Chem 2005; 280: 22819-22826, doi: 10.1074/jbc.M501106200.

25. Sakoda T, Kasahara N, Hamamori Y, Kedes L. A high-titer lentiviral production system mediates efficient transduction 
of differentiated cells including beating cardiac myocytes. J Mol Cell Cardiol 1999; 31: 2037-2047, doi: 10.1006/jmcc. 1999.1035.

26. Zhang $\mathrm{H}$, Jiang $\mathrm{H}$, Wang $\mathrm{W}$, Gong J, Zhang $\mathrm{L}$, Chen Z, et al. Expression of Med19 in bladder cancer tissues and its role on bladder cancer cell growth. Urol Oncol 2012; 30: 920927, doi: 10.1016/j.urolonc.2010.10.003.

27. Zhang X, Wan G, Mlotshwa S, Vance V, Berger FG, Chen H, et al. Oncogenic Wip1 phosphatase is inhibited by miR-16 in the DNA damage signaling pathway. Cancer Res 2010; 70: 7176-7186, doi: 10.1158/0008-5472.CAN-10-0697.

28. Yin $\mathrm{H}$, Yan Z, Liang $\mathrm{Y}$, Liu B, Su Q. Knockdown of protein phosphatase magnesium-dependent 1 (PPM1D) through lentivirus-mediated RNA silencing inhibits colorectal carcinoma cell proliferation. Technol Cancer Res Treat 2013; 12: 537-543

29. Parssinen J, Alarmo EL, Karhu R, Kallioniemi A. PPM1D silencing by RNA interference inhibits proliferation and induces apoptosis in breast cancer cell lines with wild-type p53. Cancer Genet Cytogenet 2008; 182: 33-39, doi: 10.1016/ j.cancergencyto.2007.12.013.

30. Zhu YH, Bulavin DV. Wip1-dependent signaling pathways in health and diseases. Prog Mol Biol Transl Sci 2012; 106: 307-325, doi: 10.1016/B978-0-12-396456-4.00001-8.

31. Demidov ON, Kek C, Shreeram S, Timofeev O, Fornace AJ, Appella $\mathrm{E}$, et al. The role of the MKK6/p38 MAPK pathway in Wip1-dependent regulation of ErbB2-driven mammary gland tumorigenesis. Oncogene 2007; 26: 2502-2506, doi: 10.1038/sj.onc. 1210032 . 